\title{
A Short Review and a Comparison of Simulation Models of Electrical Treeing Development in Solid Insulation
}

\author{
C. Satrazanis ${ }^{1}$, N. C. Mavrikakis ${ }^{2, *}$, K. G. Siderakis ${ }^{2}$ and M. G. Danikas ${ }^{1}$ \\ ${ }^{1}$ Department of Electrical and Computer Engineering, Democritus University of Thrace University, Xanthi, Greece \\ ${ }^{2}$ Department of Electrical and Computer Engineering, Hellenic Mediterranean University, Heraklion, Greece
}

Received 13 May 2020; Accepted 21 August 2020

\begin{abstract}
The reliability of high voltage insulation systems is significantly affected by the electrical treeing, thus the physical mechanisms interpreted this phenomenon have attracted the interest of many researchers. In this direction, several significant simulation models have been proposed to determine the impact of critical factors on the initiation and the development of the electrical treeing. In this paper, a short review of the most known and widely accepted simulation models of electrical treeing as well as a comparison of them is reported.
\end{abstract}

Keywords: electrical treeing, fractals, space charge, insulation deterioration, insulation degradation

\section{Introduction}

Solid insulating materials demonstrate increased levels of dielectric strength when compared to gas and liquid dielectrics, providing the capability of reducing size of high voltage system while maintaining increased operating voltage. Also, considering that the mechanical strength of gas and liquid dielectrics is exploited to undertaking load, solid dielectrics are necessary to ensure the safety distancesdielectric gaps in high voltage systems. Typical examples of the latter are spacers and outdoor insulators [1].

On the other hand, the incapability of dielectric recovery following breakdown is the primary disadvantage of solid dielectrics. There is still the need to better understand the mechanism of solid dielectric breakdown, that concludes to the insulation failure, under the applied stress. Other disadvantages can be noted, such as the relatively high dielectric losses $\left(\tan \delta>10^{-3}\right)$ and the low thermal conductivity of solid insulating materials [2].

Furthermore, the dielectric strength of solids is significantly affected by material imperfections associated with the presence of cavities and foreign particles and the formation of interfaces between particles of different materials, as well as by environmental conditions, such as temperature [3-5].

A pre-breakdown phenomenon, strongly affecting the dielectric strength of solid insulation is electrical treeing. It refers to the formation of electrical paths, consisted of irregularly interconnected channels that propagate through the solid material, under the application of electrical stress. The channels' growth looks like a microscopic tree, thus this phenomenon is called treeing. This formation, is closely related to partial discharges activity [6] and may lead to insulation breakdown. Treeing mainly initiates from the areas of enhanced electric field, that is enclosed cavities or foreign particles, which exist in the volume of the solid insulation.

*E-mail address: nmavrikakis@hmu.gr

ISSN: $1791-2377$ @ 2020 School of Science, IHU. All rights reserved.

doi:10.25103/jestr.134.05
The latter is true for the industrial solid insulation materials $[6,7]$.

The propagation of electrical treeing is mainly affected by the applied voltage, the composition of the insulating material and the service/experimental conditions. Treeing is favoured under extremely non uniform electric fields; and therefore laboratory studies incorporate the point-plane electrode arrangement. Further treeing development is more evinced under ac voltage, but it can be also observed when applying dc voltage. Under ac voltage, the development is attributed to the back and forth injection and extraction of electrons, from the point electrode, during the negative and the positive halfcycles respectively. Some of the electrons may acquire enough energy to decompose the material, resulting in the formation of byproducts. The electrochemical changes occur in the molecular structure of the material, are associated with the formation of conductive channels, contributing to further development of partial discharges [8].

Several models have been proposed, aiming to provide a physical explanation of the electrical tree propagation. Among them, the model of local thermal deterioration [9], the model of electromechanical pressure [10], the model of deterioration due to partial discharges activity [11] and the model of degradation of the solid insulation due to the injection and extraction of electrons [12] are mostly known. In addition to the physical explanation of electrical treeing, research work also incorporates simulation studies, in order to parameterize the physical factors of the phenomenon and to investigate their impact on the propagation of electrical treeing.

In this paper a review of the most known and widely accepted proposed models is presented aiming to evaluate the advantages and weaknesses of each one.

\section{Electrical Treeing Models}

Inhomogeneities in the solid dielectric material volume in combination with the applied voltage stress, is the cause of 
electrical treeing. These inhomogeneities are formed due to the presence of fillers and additives, sizing from a few nanometers up to $\sim 100 \mu \mathrm{m}$. The purpose of these particles is to form the desired material properties. However, when they are not sufficiently incorporated in the material structure, the interfaces formed between solid insulation material and particles, may cause morphological deterioration of the material, such as fissures, defoliations or micro-cracks [13], which favours the initiation of electrical treeing. Furthermore, electrical treeing may also occur due to the enclosed cavities in a solid dielectric, formed within manufacturing process as a result of imperfections [14]. In any case it has to be noted that the electrical treeing is intimately related to the partial discharges [15].

Simulation studies of electrical treeing, significantly contribute to better understand this phenomenon. However, although simulations are useful tools, they hardly represent the principles of a physical phenomenon because only some of the actual factors are considered. In the following paragraphs, some of the most known simulation models are described and compared.

\subsection{The Niemeyer-Pietronero-Wiesmann Model}

The Niemeyer-Pietronero-Wiesmann model, widely known as NPW model, is the first stochastic two-dimensional model developed in 1984 by the ABB research group. This model tries to reproduce the pattern of streamers and leaders propagation in dielectrics, emphasizing to the fractal characteristics of the process. According to this model, the probability of tree development depends on the local electric field and a factor which determines the randomness of the process. The model was used to facilitate the analysis of an experimental discharge, formed on the surface of a glass, surrounded by $\mathrm{SF}_{6}$ at a pressure of $0.3 \mathrm{MPa}$, by implementing a non-uniform electric field arrangement and applying a short time high voltage. The simulation results were in good agreement with the experimental surface discharge pattern, indicating the fractal character of the process [16].

The simulation requires a two-dimensional mesh. The central point of the mesh represents the point of contact of the sharp electrode with the solid dielectric. The potential at this point is considered to be $\varphi=0$. The second electrode is represented as a circle with a large radius and with a potential $\varphi=1$. The model creates a trace, which represents the path of the discharge (Fig.1). The tracing of the breakdown develops in discrete steps and - with every repetition of the process - a point is added. The potential is calculated in every point according to Laplace's equation with the aforementioned boundary conditions for $\varphi$.

For each possible direction of the treeing propagation a possibility is calculated, which is a function of the potential difference between the points of tracing $(i, k)$ and the adjacent points (i', k'). The possibility of propagation in each direction is calculated, according to equation (1):

$$
p\left(i, k \rightarrow i^{\prime}, k^{\prime}\right)=\frac{\left(\phi_{i^{\prime}, k^{\prime}}\right)^{\eta}}{\sum\left(\phi_{i^{\prime}, k^{\prime}}\right)^{\eta}}
$$

Where, $\varphi$ is the potential difference between the points (i, $\mathrm{k}$ ) and (i', k') and $\eta$ is a constant value ranging within $[0,1]$, associated with the relation of the local electric field with the possibility of discharge propagation.

The point with the highest possibility is added to the tracing path. Further a potential $\varphi=0$ is assumed and then the potential of all points is recalculated according to the Laplace equation:

$$
\nabla^{2} \phi=0
$$

Although this model may be considered as a breakthrough in the field, there are two major drawbacks that have to be considered. Firstly, in the calculation process, the time dependence of the discharge propagation is neglected. Furthermore, a threshold value for the voltage or the electric field is not considered in the model, thus implying that the electrical treeing occurs even at very low values of voltage (or electric field).

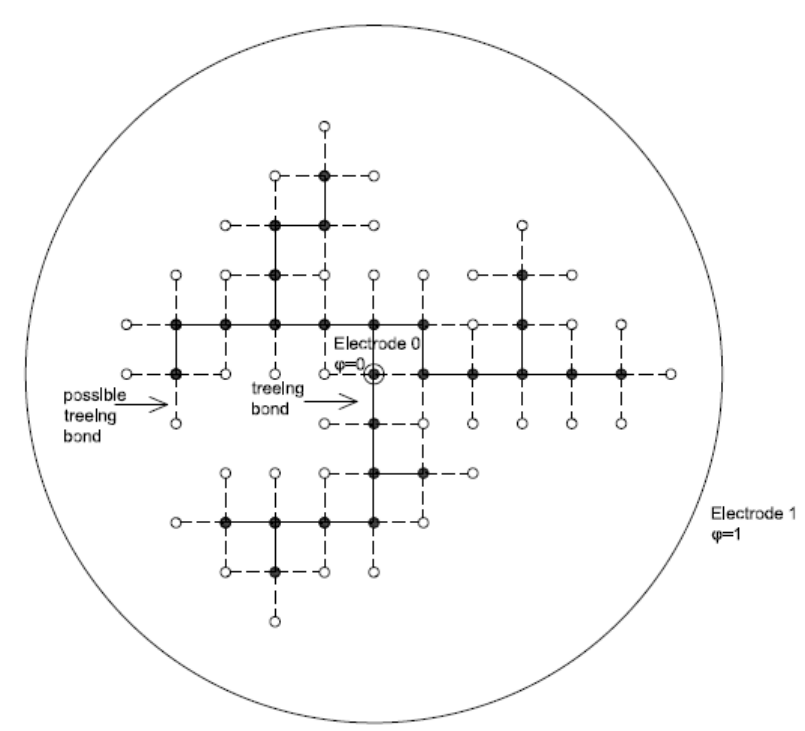

Fig. 1. Estimation of discharge pattern according to NPW model [16]. 2.2 The Wiesmann-Zeller Model

The Wiesmann and Zeller (WZ) model [17] presents an effort to correct the drawbacks of the NPW model. Their model is a generalization of NPW model and was developed by considering that the electrical treeing propagates along a needle plate electrode geometry. The basic fundamental concepts for the discharge propagation are the same as in the NPW model. In their calculations, Wiesmann and Zeller assumed that the potential at the point electrode was $\varphi=0$ and the potential at the plane electrode $\varphi=\varphi_{0}$. It was also considered that the potential $\varphi=0$ is true for all points which consist part of the tree. It was assumed that there was a twodimensional mesh of points between the two electrodes. In each repetition of the calculation, a new point was added to the tree based on the probability equation of (1). The significant difference between the WZ model and the NPW model is that the probability of tree propagation depends on the local value of the electric field $\left(\mathrm{E}_{\mathrm{loc}}\right)$. Wiesmann and Zeller introduced two parameters, deterministic for their model, namely the critical field $\mathrm{Ec}$ and the internal field $\mathrm{E}_{\mathrm{s}}$. The critical field $E_{c}$ is the threshold field which is required for the tree propagation, which means that equation 1 is true only if the local value of the electric field $\left(\mathrm{E}_{\mathrm{loc}}\right)$ is higher than that of the critical field $\left(\mathrm{E}_{\mathrm{c}}\right)$, otherwise the probability of tree propagation is zero. The other parameter is the internal field in the structure $E_{s}$. Based on this assumption, the potential in every point of the discharge structure is calculated by the equation:

$$
\phi=\phi_{0}+s \cdot E_{s}
$$


where, $\varphi_{0}$ is the potential of the plate electrode and $s$ is the length of the path which connects the point with the needle electrode.

The simulations, according to the WZ model, indicated that the shape and the form of the resulting simulated tree are determined by the critical electric field and the voltage drop across the bonds. It was observed that by increasing the values of both aforementioned quantities, the number of bonds of the tree structure was reduced. Also, as shown in Figure 2, more stable partial discharge structure and an increase of branching are occurred with the increase of the ratio $E_{s} / E_{c}$. The novelty of the WZ model was the introduction of the notion of critical electric field as well as the notion of the voltage drop across the bonds. The time factor, however, was again absent from this model.
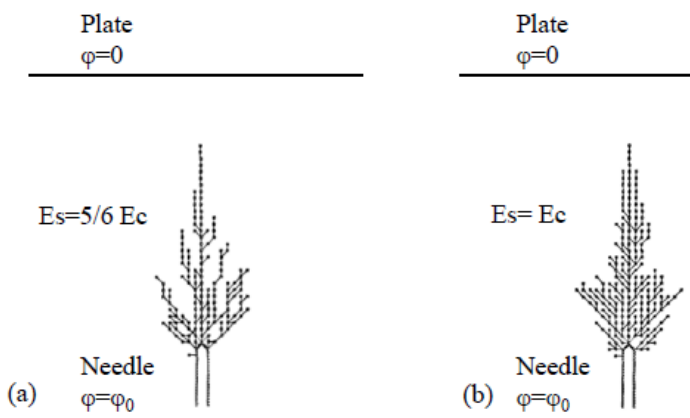

Fig. 2. Discharge structure according to $\mathrm{WZ}$ model [17], (a) Es=5/6 Ec and (b) $E s=E c$.

\subsection{The Biller Model}

The novelty of this model is the introduction of time, which is ignored both in NPW and WZ models, in a stochastic model. In Biller's model [18], the bond growth is considered as a process, which is based on the Poisson equation and the bond growth time is calculated according to the following equation:

$$
t_{i}=-\log (\xi) / r\left(E_{i}\right)
$$

where, $\xi$ is a random variable number uniformly distributed in the closed interval $[0,1]$ and $r\left(E_{i}\right)$ is a function of the local electric field, which is defined as "field dependent growth rate function". At the end of each repetition of the simulation, the lowest bond growth time is calculated. This value is considered as time step, and the associated bond is added to the tree structure. In other words,

$$
t=\min \left\{t_{i}\right\}
$$

$r\left(E_{i}\right)$ is a power law function

$$
r\left(E_{i}\right) \infty\left(E_{i} / E_{0}\right)^{n}
$$

where, $\mathrm{n}>0$ and is associated to the growth rate, $\mathrm{E}_{\mathrm{i}}$ is the local electric field and $\mathrm{E}_{0}$ is the average electric field

$$
E_{0}=U / d
$$

where, $\mathrm{d}$ is the distance between the electrodes and $\mathrm{U}$ is the applied voltage. From Figure 3, an increase of the growth rate constant $\mathrm{n}$ leads to less branches in the discharge structure.

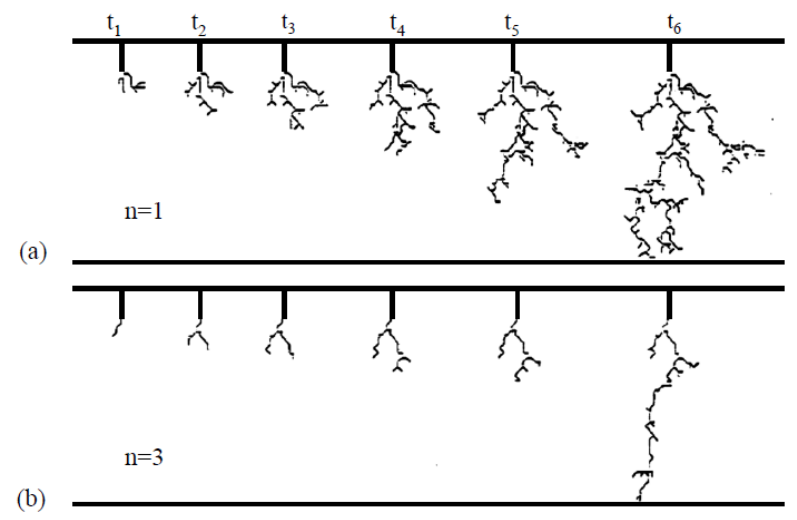

Fig. 3. Discharge structure growth with the increase of time according to Biller model [18], for (a) $n=1$ and (b) $n=3$.

\subsection{The Kupershtokh Model}

According to the Kupershtokh model, a new treeing bond is formed towards each possible direction under the assumption that the following equation is valid,

$$
E_{i}>E^{*}-\delta
$$

where, $\mathrm{E}_{\mathrm{i}}$ is the local electric field in $\mathrm{kV} / \mathrm{cm}, \mathrm{E}^{*}$ is a parameter depending on the nature of the dielectric and $\delta$ a random variable number in $\mathrm{kV} / \mathrm{cm}$ [19]. The quantity of $\left(\mathrm{E}^{*}-\delta\right)$ is characteristic for all the random factors, which may affect the streamer propagation, such as local inhomogeneities of the dielectric, cosmic radiation, temperature, humidity etc. The probability of adding a new bond to the tree structure - during a defined time step - can be given as

$$
p\left(E_{i}\right)=\int_{E^{*}-E_{i}}^{\infty} f(\delta) d \delta
$$

with $\delta>E^{*}-E_{i}$. Similarly to NPW and WZ models, this model ignores the time factor and the parameter $E^{*}$ is not well defined.

\subsection{The Noskov Model}

This model tries to relate the partial discharge (PD) activity with the treeing propagation [20]. According to this model, the PD activity is related to the length and the number of channels of electrical tree structure. The distribution of the electric field and the deterioration around the channels were taken into account. An important parameter in this model, besides the critical field $\mathrm{E}_{\mathrm{c}}$, the threshold ignition field $\mathrm{E}_{\mathrm{ign}}$, the residual field $\mathrm{E}_{\mathrm{res}}$, and bond length $\mathrm{d}$, was the threshold specific energy $\mathrm{W}_{\mathrm{c}}$. The dielectric material deterioration associated with the PD activity within the tree channels and the diffused energy caused by PD. New channels are formed when the local deterioration reaches a critical value and the local electric field near the region - which underwent the deterioration - surpasses a critical value. This model, indeed takes into account a number of parameters, assumes that the charge transport takes place only during PD events. This is a weakness recognized by the authors of [20].

\subsection{The Dodd Model}

Dodd's model is based on electrostatics and the relation of electron avalanches and PD activity with the insulation 
damage [21]. In this model, only the tree segments where partial discharges had been developed at least once would be part of the tree structure. The damaged segments could support discharges, resulting further to the propagation of the tree structure. Thus, tree bonds are formed if a discharge occurs along such segments. For the formation of damaged segments, there are threshold values for the diffused energy and the local electric field. This model was one of the most significant approaches for creating a deterministic model. However, this model does not consider vital factors affecting the tree structure such as temperature and pressure. The model is based on two fundamental routines as shown in Figure 4, one for partial discharge process and one for the material damage.

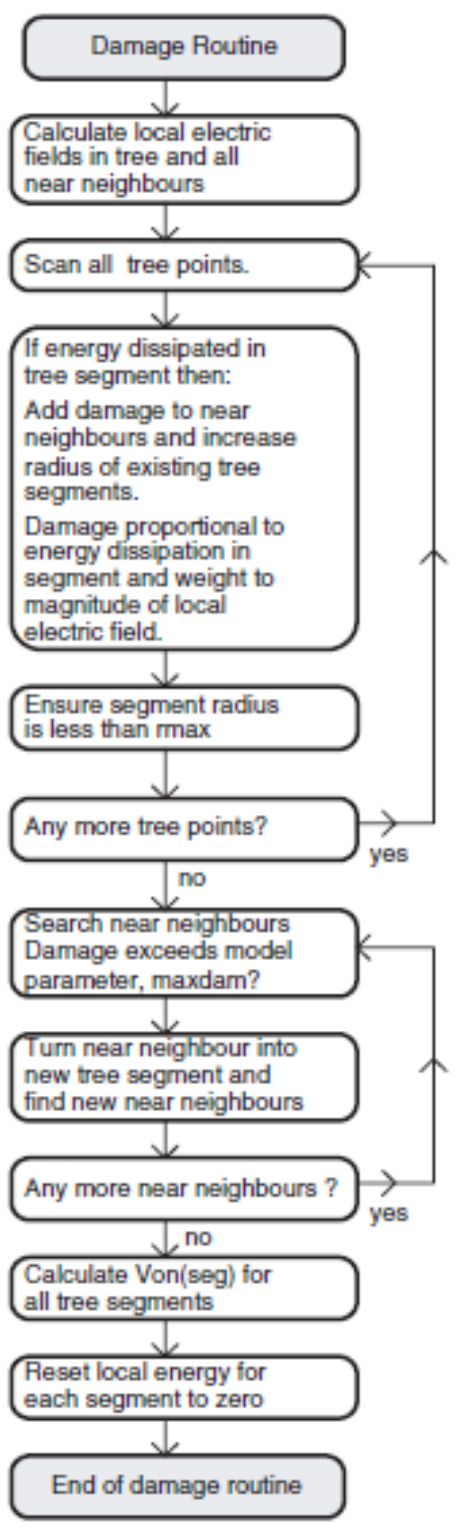

Fig. 4. Partial discharge routine and material damage routine according to Dodd model [21].

\subsection{The Petrov-Petrova-D'alessandro Model}

This model has been developed in order to simulate lightning strikes to structures by using the fractal approach [22]. The model is based to some extent on the NPW and WZ works. The novelty of this work is that the simulations are threedimensional. Although this model is not applied to solid dielectrics, it introduces the discharge propagation in threedimensional expansion. The probability of breakdown is taken to be proportional to a power $\eta$ of the local field in the region in front of the leader channel, i.e.

$p \sim E^{\eta}$

with $\eta>0$. Furthermore, this work offers a prediction of the polarity effect to the discharge propagation.

\subsection{The Dissado Model}

This model tries to combine the treeing development with the PD activity inside the tree channels. According to this model, the PD inside the tree channels enhance the electric field at the tip of the tree rendering thus discharge avalanches possible, which in turn cause the deterioration of the dielectric material [23]. This model gives a new insight - instead of advancing a stochastic selection of bonds to the tree structure - it introduces the quantitative damage of each bond per unit time for the channel formation. Moreover, the space charges from the previous discharges are considered for calculating the local electric field. According to this model a new bond is added to the tree structure as a consequence of the accumulation of damage under specified local conditions, the strength of the dielectric material and the local electric field. The merit of this model is that it gives a physical background to the whole process of electrical tree modelling. 


\subsection{The Chongqing Model}

This model, although it does not strictly refer to solid dielectrics, is a three-dimensional model based on the fractal approach [24]. The authors - based on observations - claim that the treeing path (in their case the lightning path) has both branches and tortuosity, which affect the electric field and therefore the progression of the tree (in their case the progression of the leader). This is a model based also on the values of the breakdown probability constant $(\eta)$. Smaller values of $\eta$ imply a more tortuous path for the tree progression (Fig.5). Although this paper is directed to phenomena related to lightning, the model proposed can be useful for treeing studies.

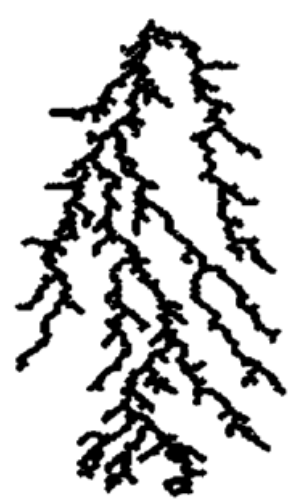

$\eta=0.1$ $\eta=1$
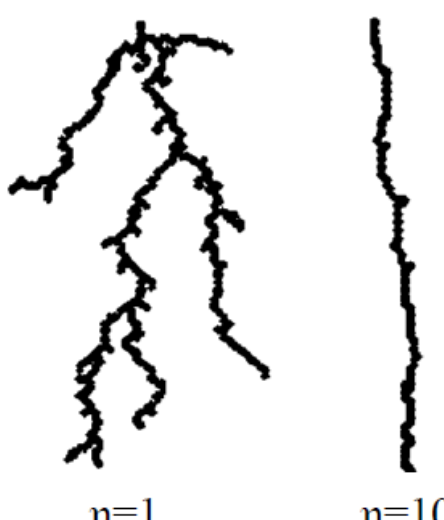

Fig. 5. Partial discharge structure according to Chongqing model [24] for different values of breakdown probability $\eta$.

\section{Discussion}

Treeing development in a solid dielectric is a complex phenomenon difficult to be modelled and thus each of the models proposed has advantages and drawbacks. There are two fundamental factors affecting the electrical treeing development. The first is the applied electric stress and the second is the condition of the dielectric (Fig.6). The electric stress is calculated implementing the known theory, for example the Laplace Equation. Therefore, there is a known and defined procedure to evaluate its contribution. On the other hand the second, i.e. the material condition, is difficult to be determined since it is correlated with material impurities and the influence of the ongoing applied stress. Thus for the second factor, either a deterministic or a stochastic approach can be implemented. Nevertheless, in both cases influencing parameters are not considered. Models based on the fractal theory ignore physical quantities, whereas the models which consider some physical quantities, ignore other factors, such mechanical stress, which may have a significant impact on the propagation of electrical trees. As it is cited in [25], an external stress factor either mechanical or electrical may locally affect the insulation level, a fact that is rather ignored in the models given above. In addition, it must be noted that most of the models ignore the time factor. Since the time required for the electrical treeing development is crucial (because time, in several cases, is closely related to the aging of the insulation), this research focuses on the factors required for the modelling of treeing propagation. A closer investigation between electrical tree modelling and experimentation could give a better understanding of tree propagation [26-28].

$\begin{array}{llll}\text { Electrical stress } & +\quad \begin{array}{l}\text { Condition of dielectric } \\ \text { Laplace equation }\end{array} & \begin{array}{l}\text { Deterministic or } \\ \text { stochastic approach for: } \\ \text { 1. Material impurities } \\ \text { 2. Effects of the applied } \\ \text { electrical stress on the } \\ \text { material }\end{array} & \begin{array}{l}\text { Estimation of } \\ \text { development }\end{array} \\ & \end{array}$

Fig. 6. Fundamental factors affecting the development of electrical treeing.

The initiation and the propagation of electrical treeing is modelled by using 2D or 3D models and by using stochastic, deterministic or fractal approaches. In accordance to the latter, the models presented in this study can be grouped as shown in Figure 7.

The introduction of probability to the simulation procedure, was a breakthrough presented by the NPW model and it was further embedded by the WZ and Biller models, which can be considered as improvements of the initial concept. Likewise the Kupershtokh model incorporates the stochastic nature of the tree development, considering different parameters for the probability calculation. At the same time deterministic models have been suggested like the Dodd model and the Dissado model, where in both cases the material condition due to the stress applied by the partial discharges is estimated in order to proceed to the tree development. The presence and intensity of partial discharges are critical factors, however, it is difficult to achieve a reliable estimation, therefore the stochastic nature of the phenomenon may still apply. The Noskov model is a combination where the presence of partial discharges is evaluated and considered, but the tree development and especially the direction of the tree development is still considered stochastic and a probability is calculated. However, in this case also, there is a weakness since although the model incorporates a number of parameters, it assumes that the charge transport only takes place only during PD events.

Further, the Petrov-Petrova-D'Alessandro and the Chongqing model were evaluated. Both are 3D models with an initial scope to simulate lightning discharges. In this case also the concept of probability is present, while incorporating in both cases the fractal approach. These models were developed for the study of lightning discharges, and although their application is limited, they can be proved useful also in the case of solid dielectric treeing.

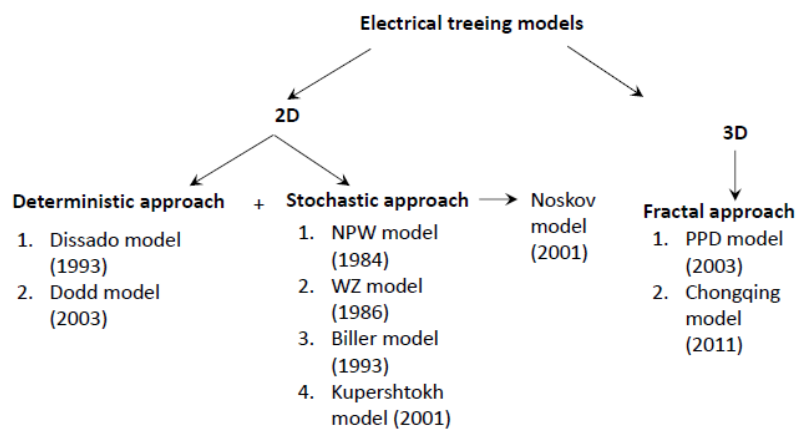

Fig. 7. Chart of electrical treeing models.

Finally the models, cited in the present paper, require to define critical values on the parameters, i.e. trees could take place if some critical values are satisfied. An aspect somehow neglected - is that even with small (rather innocuous) PD activity, treeing structures may also be 
produced. Thus, the question as to what is a critical value and what is not, is something that persists with us even today. Germs of such thoughts one may trace in [23], although in this case, the authors do not go into any length in exploring the possibilities of accumulated damage even below some critical value (of the local electric field or of some sort of critical energy which must be delivered). The questions as to whether there may be damage because of discharges and/or charging phenomena below the so-called inception voltage, may not be directly related to the models of treeing but - especially in the context of the approach of [23] - should be discussed and could be a field of further research. Several papers, tackling these aspects of possible damage below the called inception voltage, have already been published [29-31]. Furthermore, in recent studies [32-34] regarding the electrical treeing in insulating materials, the chemical and the morphological changes have been investigated in order to better understand the electrical treeing propagation. Also, the analysis of partial discharge features could be an effective tool for predicting the breakdown of insulating materials due to electrical treeing [35]. It would be a challenge for future research to try to deal with some aspects of treeing modelling, having to take into account the aforementioned publications.

\section{Conclusions}

In this short review several models, available for simulating electrical treeing phenomenon are presented. Stochastic or/and deterministic approaches have been implemented so as to consider fundamental factors for the initiation and propagation of electrical treeing. However, the simplifications considered in these models ignore the effect of physical phenomena on the electrical treeing process, such as the space charge effect, pre-breakdown discharges and the inhomogeneity of the material. It is a challenge for the future, the electrical treeing models to be combined with experimental observations and material analysis techniques so as to improve their accuracy as a predictive tool.

This is an Open Access article distributed under the terms of the Creative Commons Attribution License

\section{References}

[1] T. J. Gallagher, A. J. Pearmain, High voltage: Measurement, testing and design, New York, USA: Eds. John Wiley \& Sons, 1983.

[2] F. H. Kreuger, Industrial high voltage: Electric fields, dielectrics, constructions, Delft, The Netherlands: Eds. Delft University Press, 1991.

[3] D. H. Schroff, A. W. Stannett, "Contribution to the study of electric strength of polythene," Proceedings of IEE, vol. 114, no. 6, pp. 832 833, 1967. DOI: 10.1049/piee.1967.0161.

[4] A. L. McKean, "Breakdown mechanism studies in cross-linked polyethylene cable," IEEE Transactions on Power, Apparatus and Systems, vol. 95, no. 1, pp. 253-260, 1976. DOI: 10.1109/TPAS.1976.32099.

[5] J. H. Mason, "Assessing the resistance of polymers to electrical treeing," Proceedings of IEE A, vol. 128, no. 3, pp. 193-201, 1981. DOI: 10.1049/ip-a-1.1981.0030.

[6] W. Vahlstrom, "Investigation of insulation deterioration in $15 \mathrm{kV}$ and $22 \mathrm{kV}$ polyethylene cables removed from service," IEEE Transactions on Power, Apparatus and Systems, vol. 91, no. 3, pp. 1023-1035, 1972. DOI: 10.1109/TPAS.1972.293454.

[7] J. H. Lawson, W. Vahlstrom, "Investigation of insulation deterioration in $15 \mathrm{kV}$ and $22 \mathrm{kV}$ polyethylene cables removed from service - Part II," IEEE Transactions on Power, Apparatus and Systems, vol. 92, no. 2, pp. 824-835, 1973. DOI: 10.1109/TPAS.1973.293790.

[8] T. Tanaka, A. Greenwood, "Effects of charge injection and extraction on tree initiation in polyethylene," IEEE Transactions on Power, Apparatus and Systems, vol. 97, no. 5, pp. 1749-1759, 1978. DOI: 10.1109/TPAS.1978.354668.

[9] R. Cooper, "Breakdown in solids", in Electrical Insulation, A. Bradwell, London, UK: Eds. Peter Peregrinus Ltd., 1983, pp. 33-51.

[10] M. Ieda, M. Nawata, "A consideration of treeing in polymers," 1972 Annual Report on Conference of Electrical Insulation and Dielectric Phenomena, National Academy of Sciences, Washington, D. C., USA, pp. 143-150, 1973. DOI: 10.1109/CEIDP.1972.7734158.

[11] C. Mayoux, "On the degradation of insulating materials withstanding electrical Stresses," 2000 Annual report on Conference of Electrical Insulation and Dielectric Phenomena, Victoria, B. C., Canada, pp. 1-20, 2000. DOI: 10.1109/CEIDP.2000.885217.

[12] K. C. Kao, De Min Tu, "Formation of electrical treeing in polyethylene", 1982 Annual Report on Conference of Electrical Insulation and Dielectric Phenomena, Amherst, MA, USA, pp. 598603,1982. DOI: 10.1109/CEIDP.1982.7726585.

[13] R. Bruetsch, M. Chapman, "Insulating systems for rotating machines and reliability considerations," Proceedings of the 2010 International Symposium on Electrical Insulation, San Diego, CA, USA, Publication 978-1- 4244-6301-5/106-9, 2010. DOI: 10.1109/ELINSL.2010.5549737.
[14] K. Mueller, D. Meurer, "Auswirkungen von Teilentladungen auf elektrische Isolierstoffe,", in Teilentlandungen in Betriebsmitteln der Energietechnik, D. Koenig and Y. Narayama Rao, Berlin, Germany: Eds. VDE Verlag, pp. 85-104,1993.

[15] T. Okamoto, T. Tanaka, "Auto-correlation function of PD pulses under electrical treeing degradation," IEEE Transactions on Dielectrics and Electrical Insulation, vol. 2, no. 6, pp. 857-865, 1995. DOI: 10.1109/94.469979.

[16] L. Niemeyer, L. Pietronero, H. J. Wiesmann, "Fractal dimension of dielectric breakdown," Physical Review Letters, vol. 52, no. 12, pp. 1033-1036, 1984. DOI: 10.1103/PhysRevLett.52.1033.

[17] H. J. Wiesmann, H.-R. Zeller, "A fractal model of dielectric breakdown and prebreakdown in solid dielectrics," Journal of Applied Physics, vol. 60, no. 5, pp. 1770-1773, 1986. DOI: 10.1063/1.337219.

[18] P. Biller, "Fractal streamer models with physical time," in Proc. $11^{\text {th }}$ International Conference on Breakdown in Dielectric Liquids, Baden-Daettwil, Switzerland, IEEE No. 93CH3204-6, pp. 199-203, 1993. DOI: 10.1109/ICDL.1993.593938.

[19] A. L. Kupershtokh, V. Charalambakos, D. Agoris, D. I. Karpov, "Simulation of breakdown in air using cellular automata with streamer to leader transition,” J. Phys. D.: Appl. Phys, vol. 34, no. 6, pp. 936-946, 2001. DOI: 10.1088/0022-3727/34/6/315.

[20] M. D. Noskov, M. Sack, A. S. Malinovski, A. J. Schwab, "Measurement and simulation of electric tree growth and partial discharge activity in epoxy resin," J. Phys. D.: Appl. Phys., vol. 34, no. 9, pp. 1389-1398, 2001. DOI: 10.1088/0022-3727/34/9/316.

[21] S. J. Dodd, "A deterministic model for the growth of non-conducting electrical tree structures," J. Phys. D.: Appl. Phys., vol. 36, pp. 129141, 2003. DOI: 10.1088/0022-3727/36/2/309.

[22] N. I. Petrov, G. N. Petrova, F. D’Alessandro, “Quantification of the probability of lightning strikes to structures using a fractal approach", IEEE Transactions Dielectrics and Electrical Insulation, vol. 10, no. 4, pp. 641-654, 2003. DOI: 10.1109/TDEI.2003.1219649

[23] L. A. Dissado, P. J. Sweeney, "Physical model for breakdown structures in solid dielectrics," Physical Review B, vol. 48, no. 22, pp. 16261-16268, 1993. DOI: 10.1103/PhysRevB.48.16261.

[24] J. Li, Q. Yang, W. Sima, C. Sun, T. Yuan, M. Zahn, "A new estimation model of the lightning shielding performance of transmission lines using a fractal approach," IEEE Transactions on Dielectrics and Electrical Insulation, vol. 18, no. 5, pp. 1712-1723, 2011. DOI: 10.1109/TDEI.2011.6032843.

[25] G. E. Vardakis, "Breakdown phenomena in solid insulating materials: A study of Electrical tree propagation", $\mathrm{Ph}$. D. dissertation, Democritus University of Thrace, Department of Electrical and Computer Engineering, Xanthi, Greece, 2006 (in Greek). 
[26] T. Hibma, H. R. Zeller, "Direct measurement of space charge injection from a needle electrode into dielectrics," Journal of Applied Physics, vol. 59, no. 5, pp. 1614-1620, 1985. DOI: 10.1063/1.336473.

[27] H. R. Zeller, W. R. Schneider, "Electrofracture mechanics of dielectric aging," Journal of Applied Physics, vol. 56, no. 2, pp. 455 459, 1984. DOI: 10.1063/1.333931.

[28] H. R. Zeller, "Breakdown and prebreakdown phenomena in solid dielectrics," IEEE Transactions on Electrical Insulation, vol. 22, no. 2, pp. 115-122, 1987. DOI: 10.1109/TEI.1987.298867.

[29] A. M. Bruning, D. G. Kasture, F. J. Campbell, N. H. Turner, "Effect of cavity sub-corona current on polymer insulation life," IEEE Transactions on Electrical Insulation, vol. 26, no. 4, pp. 826-836, 1991. DOI: 10.1109/14.83709.

[30] A. M. Bruning, M. G. Danikas, "Experiments on polymer cavity currents above and below CIV", 1992 Annual report on Conference of Electrical Insulation and Dielectric Phenomena, Victoria, B.C. Canada, pp. 735-740, 1992. DOI: 10.1109/CEIDP.1992.283132.

[31] A. Harlin, M. G. Danikas, P. Hyvonen, "Polyolefin insulation degradation in electrical field below critical inception voltage", Journal of Electrical Engineering, vol. 56, no. 5, pp. 135-140, 2005.
[32] S.M. Rowland, R. Schurch, M. Pattouras, Q. Li, "Application of FEA to image-based models of electrical trees with uniform conductivity", IEEE Transactions on Dielectrics and Electrical Insulation, vol. 22, no. 3, pp. 1537-1546, 2015. DOI:10.1109/TDEI.2015.004922.

[33] Y. Liu, X. Cao, "Electrical tree growth characteristics in XLPE cable insulation under DC voltage conditions", IEEE Transactions on Dielectrics and Electrical Insulation,, vol. 22, no. 6, pp. 3676-3684, 2015. DOI: 10.1109/TDEI.2015.005222.

[34] S. Iwata, "Influence of humidity treatment on electrical tree propagation in epoxy resin", IEEE Transactions on Dielectrics and Electrical Insulation,, vol. 23, no. 5, pp. 2556-2561, 2016. DOI: 10.1109/TDEI.2016.7736812.

[35] N.H. Aziz, V.M. Catterson, S.M. Rowland, S. Bahadoorsingh, "Analysis of partial discharge features as prognostic indicators of electrical treeing", IEEE Transactions on Dielectrics and Electrical Insulation, vol. 24, no. 1, pp. 129-136, 2017. DOI: 10.1109/TDEI.2016.005957. 